\title{
In-situ XRPD study of ammonolysis of cobalt-molybdenum ammonia synthesis catalysts with defined Co to Mo ratio
}

\author{
P. Adamski, A. Albrecht, D. Moszyński \\ West Pomeranian University of Technology in Szczecin, Faculty of Chemical Technology and Engineering, Department of Chemical \\ and Environment Engineering, Putaskiego 10, 70-322 Szczecin, Poland \\ adamski_pawel@zut.edu.pl
}

Ternary transition metals nitrides are a relatively new group of materials studied extensively nowadays. One of the most important properties of ternary transition metals nitrides is their tendency to form defected structures, with variable composition. Within these inorganic compounds, the Co-Mo-N system exhibits many promising properties. Among others, cobalt molybdenum nitrides could be used as catalysts, magnetic materials and electrodes. Cobalt molybdenum nitrides exhibit very high catalytic activity in ammonia synthesis, which makes them a plausible candidate to replace industrial iron catalyst [1-3].

The most widely used procedure to form cobalt molybdenum nitrides is a two-stage process consisting of a precursor preparation and a subsequent ammonolysis of mixed oxides. More insight into these stages, especially on structural and crystallographic transformations of precursors and intermediate compounds, is crucial for the enhancement of the material properties. The synthesis process is influenced by numerous parameters, i.e. composition and temperature of precipitation process, final precursor composition, ammonolysis temperature, composition and flow of a reducing agent. Consequently, the ammonolysis often results in the formation of mixtures of different crystallographic phases of transition metal nitrides with mismatched properties. Lack of reproducibility is a major disadvantage, which inhibits technology upscaling.

A synthesis method of cobalt molybdenum nitrides greatly affects its composition and properties. The stoichiometry alteration could be beneficial or detrimental to the cobalt molybdenum nitrides properties. For example, their catalytic activity in ammonia synthesis depends on $\mathrm{Co}_{2} \mathrm{Mo}_{3} \mathrm{~N}$ to $\mathrm{Co}_{3} \mathrm{Mo}_{3} \mathrm{~N}$ ratio [4]. Therefore, favourable is a synthesis method, which can affect stoichiometry in a controlled way. Such a procedure is the one used in this study. It bases on the mechanochemical formation of the mixture of cobalt and molybdenum salts with the controlled Co:Mo molar ratio, which is later reduced under the ammonia atmosphere.

To make insight into the activation process, the ammonolysis of the mixture of cobalt and molybdenum salts with the controlled Co:Mo molar ratio was examined via in-situ X-ray powder diffraction with the use of PANalytical X'pert Pro MPD diffractometer equipped with Anton Paar XRK 900 reaction chamber. A transformation of mixed cobalt molybdenum oxides into mixed cobalt molybdenum nitrides was observed. In the sample subjected to the temperature of $200^{\circ} \mathrm{C}$ the reflections corresponding to cobalt molybdate $\mathrm{CoMoO}_{4}$ were identified. Instead of a separate step of precursor precipitation, a simple mechanochemical technique was implemented. As a result, the phase described as the precursor in the mentioned earlier synthesis methods was obtained. This result suggests that in the studied system the intermix of cobalt and molybdenum atoms, obtained via the mechanochemical method, allows the formation of a bimetallic phase at medium temperature. In the sample at $700^{\circ} \mathrm{C}$ under an ammonia atmosphere, the reflections corresponding to $\mathrm{Co}_{2} \mathrm{Mo}_{3} \mathrm{~N}$ and $\mathrm{Co}_{3} \mathrm{Mo}_{3} \mathrm{~N}$ were identified.

[1] Jacobsen, C.J.H. (2000). Chem. Commun. 1057.

[2] Kojima, R. \& Aika, K. (2001). Appl. Catal. A 215, 149.

[3] Moszyński, D., Jędrzejewski, R., Ziebro, J. \& Arabczyk, W. (2010). Appl. Surf. Sci. 256, 5581.

[4] Moszyński, D., Adamski, P., Nadziejko, M., Komorowska, A. \& Sarnecki, A. (2018). Chemical Papers 72, 425.

\section{Keywords: Cobalt molybdenum nitrides; ammonolysis; XRPD in-situ}

Financed as a part of PROM Programme "International Scholarship Exchange of PhD Candidates and Academic Staff" co-financed by Polish National Agency For Academic Exchange and European Union through European Social Fund within the frame of Knowledge, Education, Development Operational Programme, project no. PPI/PRO/2019/1/00008/U/00001. 